\title{
El papel de la formación de personal en el proceso de implantación de un sistema de calidad total
}

\author{
Ignacio Danvila del Valle* \\ Miguel Ángel Sastre Castillo**
}

\section{Resumen}

Este trabajo analiza el modelo de gestión de la calidad total, haciendo esencial hincapié en las etapas necesarias para el desarrollo del sistema, concretamente en la etapa de implantación, en la que destaca la importancia de las políticas de recursos humanos elegidas por las organizaciones para buscar el compromiso y apoyo de todo el personal.

Dentro de las políticas se ha destacado la formación de personal, estableciendo como hipótesis la existencia de relación entre ésta y el sistema de calidad total.

Para el contraste de la hipótesis formulada, se ha elegido el sector de las empresas de seguridad privada existentes en España, dedicadas a la vigilancia, con una muestra de 40 empresas para las que se ha obtenido un total de 200 observaciones.

Palabras clave: calidad total, recursos humanos, formación de personal, resultados empresariales.

Fecha de recepción: 4-08-2006

Fecha de aceptación: 11-01-2007

The Role of Training in the Process of Establishment of a Total Quality System

\section{Abstract}

This work analyzes the model of total quality management, doing essential emphasis in the necessary phases for the development of the system, and concretely in the phase of implementation, in which emphasizes the importance of the Human Resources practices to seek the commitment and support of all the personnel.

\footnotetext{
* Profesor de Organización de Empresas del Centro Universitario Villanueva y de la Universidad Complutense de Madrid. Correo electrónico: idanvila@villanueva.edu

** Profesor de Organización de Empresas de la Universidad Complutense de Madrid y del Instituto Tecnológico Autónomo de México ITAM. Correo electrónico: masastre@emp.ucm.es
} 
Among the practices has itself noticeable the training of the personnel, establishing as hypothesis the existence of relation between this and the system of total quality.

For the contrast of the hypothesis formulated has been choosed the sector of private security firms dedicated to the caution, existing in Spain, with a sample of 40 firms, and a total of 200 observations has been obtained.

Keywords: Total Quality, Human Resources, Training, Performance.

\section{Calidad total: Concepto, enfoques y etapas}

Existen numerosas definiciones del término calidad, las cuales han sido propuestas por los principales expertos en el estudio de esta materia. Muchas de estas definiciones pueden ser consideradas complementarias entre sí y aparecen recogidas en la tabla 1.

Tabla 1

Algunas definiciones de referencia sobre el concepto de calidad

\begin{tabular}{cl}
\hline AUTOR & \multicolumn{1}{c}{ DEFINICIÓN } \\
\hline Crosby (1989) & Cumplimiento de unas especificaciones dadas. \\
Deming (1989) & Un grado predecible de uniformidad y fiabilidad a \\
& bajo coste, adecuado a las necesidades del mercado. \\
Drucker (1992) & Lo que el cliente está dispuesto a pagar en función de \\
& lo que obtiene y valora. \\
Feigenbaum (1986) & Un sistema eficaz para integrar los esfuerzos de me- \\
& jora de la calidad de los distintos grupos de una orga- \\
& nización, para proporcionar productos y servicios a \\
& niveles que permitan la satisfacción del cliente. \\
Adecuación para el uso, satisfaciendo las necesidades & \\
Tel cliente. & Pérdidas minimas para la sociedad en la vida del producto. \\
\end{tabular}

Fuente: Elaboración propia.

Con el paso del tiempo, los conceptos de calidad y control de calidad han sido modificados por el fenómeno de la calidad total. Deming (1989) dio un salto cualitativo en el estudio de la materia al pasar del concepto de calidad o control de la calidad al nuevo término de calidad total, que es el utilizado en la actualidad por las organizaciones.

El concepto de calidad total considera a la empresa como una cadena de relaciones suministrador-cliente; de esta forma cada individuo es cliente de la persona que le suministra información o material para realizar su trabajo y suministrador de la persona a la que entrega el trabajo realizado, lo que conlleva un enfoque o proceso hacia la mejora continua de la calidad. Asimismo, supone una generalización de la calidad en el sentido de aplicarse no sólo al producto final, sino también a los productos intermedios, 
a los servicios y a las relaciones entre servicios y personas. Se extiende igualmente a los distintos componentes de la empresa, a la relación entre cliente y proveedor y al empleo de determinados procedimientos para evaluar las actividades a todos los niveles. En resumen, la calidad total se extiende a todos los factores, productos y servicios, a los procesos productivos y de gestión, así como a los recursos técnicos y humanos (Galgano, 1993).

\subsection{Enfoques en la gestión de la calidad}

A partir de la noción de calidad total se desarrollan simultáneamente dos enfoques en la gestión de la misma: uno norteamericano y otro japonés.

a) El enfoque norteamericano, debido a Crosby, Juran y Feigenbaum, puede sintetizarse en los siguientes principios:

- La conformidad con las exigencias, la cual debe ser entendida como definir las exigencias por respetar, dar al personal los medios para respetarlas y dedicar todo el tiempo necesario a incitar y ayudar al personal a hacerlo.

- La prevención de los defectos. Buscar las causas de los errores y poner en marcha dispositivos preventivos. El control estadístico constituye un buen instrumento para ello.

- Criterio "cero defectos", es decir, lograr una calidad perfecta, partiendo de que los errores pueden evitarse y de que las cosas siempre se deben tratar de hacer lo mejor posible.

- Medir la calidad. El coste de la calidad comprende el coste de la no calidad (costes derivados de los errores cometidos) y el coste de la calidad (costes del sistema de conformidad necesarios para llegar a cero defectos).

b) EI enfoque japonés, cuya principal figura es Ishikawa, parte de la base de que la gestión de la calidad debe llevarse a cabo mediante la participación de todas las personas y servicios, en contraste con la idea de "especialistas de la calidad" del enfoque norteamericano. Los puntos principales del enfoque son (Ishikawa, 1985):

- La práctica de la calidad por todos los miembros de la empresa, por lo que es primordial el papel desarrollado en los niveles operativos.

- La formación para todos con el fin de lograr que la gestión de la calidad sea correctamente ejercida.

- Empleo de círculos de calidad, que son importantes pero insuficientes si no se inscriben en un planteamiento global de la calidad.

- Utilización, como instrumento, del control estadístico de la calidad.

- El establecimiento de una relación de confianza con el cliente, cuyas exigencias constituyen el objetivo esencial para la empresa. 
Con una visión integradora de ambos enfoques, Pérez Fernández de Velasco (1994: 87) aporta una interesante definición de calidad total que es concebida como "un sistema de gestión de calidad que persigue la satisfacción total de los clientes a través de la mejora continua de la calidad, de todos los métodos y procesos operativos, mediante la participación activa de todo el personal en grupos de mejora y círculos de calidad que previamente han recibido formación y entrenamiento".

En la definición anterior se destaca la idea de la participación de las personas y la trascendencia de la formación para que el sistema pueda tener éxito.

\subsection{Etapas de un modelo de gestión de calidad total}

Un modelo de gestión o metodología de implantación de un sistema de calidad total se basa en cuatro etapas básicas: etapa de diagnóstico, diseño, implantación y mantenimiento o seguimiento (Alonso y Blanco, 1990).

a) Etapa de diagnóstico. En esta etapa se pretende comprender la utilidad y los fines del modelo que deseamos desarrollar; analizar la cultura organizativa, el clima de trabajo y la estructura de la empresa; así como realizar una aproximada evaluación de los costes necesarios para llevar a cabo la implantación del sistema en la organización (Anthony, 1997).

b) Etapa de diseño. Con esta etapa se pretende preparar el proceso de implantación de la gestión de calidad total y obtener el respaldo nítido de la dirección con el proyecto de trabajo; en ocasiones, dicha etapa se externaliza (Avery, 1997).

c) Etapa de implantación de la calidad total. La etapa central de todo el proceso es la puesta en práctica de los procesos y procedimientos de calidad total en todas las operaciones de la organización. Esta etapa es la más larga y complicada, pues en primer lugar se deben crear los órganos necesarios para la implantación; después, buscar el compromiso y apoyo de todo el personal, lo cual se logra mediante adecuadas políticas de recursos humanos, las que deben incluir la formación de personal (objeto de nuestro estudio) y la adecuada comunicación e información entre todas las áreas de la empresa; por último, gestionar la mejora continua a través de técnicas operativas de gestión de calidad. (Dean y Evans, 1994).

La formación de personal es un factor esencial; por ello, la empresa debe darle su debida importancia, ya que el espíritu que subyace en la calidad total es la adopción de una actitud de aprendizaje continuo por parte de todo el personal como mejor garantía de respuesta a la necesaria mejora continua de la competitividad imprescindible para afrontar los cambios en el entorno empresarial (Brocha y Brocha, 1992). 
Esta fase debe estar perfectamente programada y debidamente planificada en el desarrollo general del proyecto. Los programas de formación y entrenamiento que se tengan deben ser adaptados a los diferentes niveles profesionales y sobre todo a la responsabilidad que cada empleado tendrá en la gestión de calidad total (Ishikawa, 1989); además, debe abarcar tanto la formación técnica como la más genérica para que permita conocer mejor el funcionamiento del sistema y la actividad global de la empresa (Aguilar y Sastre, 1998).

d) Etapa de mantenimiento o seguimiento. Esta última etapa pretende auditar el sistema de calidad total implantado en la organización, a través de un análisis económico-financiero y del estudio de los indicadores que presentan los resultados obtenidos (Arter, 1993).

\section{Calidad total y formación de personal}

Numerosos autores (Ishikawa, 1985 y 1989; Shaw, 1990; Saderra Jorba, 1994; Drummond, 1994; Im, Hartman y Bondi, 1994; Jacobs, 1995; McDuffie y Kochan, 1995; Laboucheix, 1996; Lloréns, 1996; Oppenheim y Przasnyski, 1999; Waddell y Stewart, 2004; Testa y Sipe, 2006) sostienen que la formación de personal es un elemento clave a la hora de implantar un sistema de calidad total en una organización.

De hecho, la idea de la importancia de la formación ha estado presente en todo el proceso de desarrollo de la filosofía de la calidad total. En 1967, en el Séptimo Simposio sobre Control de Calidad, celebrado en Hakone (Japón), se identificaron seis características propias del control de calidad: la participación e implicación de todos los empleados, la educación y formación en el control de calidad, las actividades de los círculos de calidad, las auditorías de calidad, el uso de métodos estadísticos y las campañas nacionales de promoción de calidad (Ishikawa, 1989).

Se puede observar que todas estas características giran en torno a los recursos humanos. En dicho congreso se considera explícitamente que el control de calidad empieza con formación y termina con formación; asimismo, si deseamos promover la calidad total deberá ser siempre con la participación de todos, es decir, se debe dar educación y formación a todos los empleados.

En la clausura de dicho congreso se llegaron a las siguientes conclusiones: "A mayor formación, mayores beneficios para los empleados y para la empresa. La empresa debe impartir formación: para cada nivel, a largo plazo, para así poder escoger su propio programa de formación y capacitación para cada empleado" (Ishikawa, 1985: 33).

En 1987, un nuevo congreso de calidad celebrado en Japón habla ya de calidad total e identifica diez características que definen este concepto. Entre éstas destacan las que hacen referencia a la formación: actividades de calidad total con participación de todos los departamentos e implicación y formación de todos los empleados (Juran, 1990). 
Igualmente, Groocock (1993) enumera una serie de rasgos para la mejora de la calidad en los programas multifuncionales entre los que destaca proporcionar la formación adecuada. Posteriormente, Saderra (1994) enumera los principios en los que se basa la implantación de un sistema de calidad total: el cliente ante todo, la mejora continua, el compromiso de la dirección, la gestión diaria de calidad en las actividades y procesos clave de la empresa, así como la participación y formación de todos los empleados.

Uno de los autores que analiza la calidad total con más profundidad es Walter Deming, quien considera la calidad total como una filosofía empresarial que se fundamenta en buscar la satisfacción del cliente. Dicha filosofía se asienta en 14 criterios (Deming, 1989), de los que dos de ellos - el número 6, "instituir la formación en el trabajo", y el número 13, "estimular la formación y el afán de superación personal" - muestran la relación existente entre la formación impartida al personal de la empresa y la calidad total.

Por último, Ishikawa (1989: 32) expresa la relación entre calidad y formación del siguiente modo: "La calidad empieza y termina con la formación. La formación ha de darse a todos los empleados, desde el presidente hasta el último trabajador. Para realizar e implantar en la organización con éxito la calidad, la formación ha de repetirse una y otra vez".

Con base en la bibliografía previa existente, que pone de manifiesto la vinculación entre sistemas de calidad total y formación, proponemos la hipótesis de nuestro trabajo.

\section{H.1.: Las empresas que poseen sistemas de gestión de calidad realizan un mayor esfuerzo en la formación de sus empleados.}

\section{Planteamiento del estudio empírico}

Con el fin de contrastar la hipótesis planteada, el estudio se ha centrado en un único sector con el objetivo de evitar problemas de heterogeneidad subyacentes al comparar efectos cuando las actividades son diversas (Black y Lynch, 2001). Otros trabajos de referencia que han seguido esta estrategia de centrarse en un único sector son Bailey (1993), Arthur (1994), Kelley (1994, 1996), Delery y Doty (1996), Dunlop y Weil (1996), así como Ichniowski, Shaw y Prennushi (1997). El sector elegido ha sido el de las empresas de seguridad privada dedicadas a la vigilancia, existentes en España. Una vez depurada, para eliminar pequeñas empresas o de reciente creación, la muestra con la que trabajamos finalmente está compuesta por 200 observaciones.

En nuestro estudio hemos tratado de profundizar en la medición de la variable formación diferenciando tres dimensiones relevantes de la misma: la variedad de la formación, el tiempo invertido por los empleados y los recursos económicos invertidos por la empresa; para ello, seguimos la metodología propuesta por Danvila y Sastre (2005). Tales dimensiones se han medido a través del número de los diferentes cursos de formación impartidos, el número de horas presenciales anuales por empleado en los cursos de 
formación recibidos y el esfuerzo inversor anual en formación que ha realizado la empresa por empleado.

Se trataba de tener un único indicador de la variable formación a partir de los datos de las tres dimensiones, ya que si fueran tomadas por separado provocarían una alta multicolinealidad y además sus interrelaciones podrían quedar ocultas. Por ello, se realizó un análisis factorial para hacer operativa la variable a la hora de contrastar la hipótesis. El método utilizado para su realización fue el análisis de componentes principales (ACP) y los resultados obtenidos son satisfactorios debido a que el factor acumula un $70 \%$ de la varianza y el porcentaje de la varianza de cada variable original explicada por el factor seleccionado es aceptable.

Siguiendo numerosos estudios (Terpstra y Rozell, 1993; George y Weimerspirch, 1994; Phillips, 1994; Saderra Jorba, 1994; Hill y Jones, 1996; Lloréns, 1996; Amat, 1998; Kroll, Wright y Heiens, 1999; Bayo y Merino, 2002) nos hemos inclinado por utilizar como variable de medición de la calidad la posesión de certificados de calidad. Los datos de esta variable han sido contrastados en los listados oficiales de entidades certificadoras. Hemos dividido las observaciones en dos grupos según posean o no certificado de calidad.

Con todo ello, nuestro objetivo consiste en contrastar si las empresas que poseen un certificado de calidad realizan un mayor esfuerzo en formación; para ello se realizará un análisis de la varianza que nos indicará si existen diferencias significativas en el esfuerzo formador de las empresas de uno y otro grupo.

\section{Resultados}

Iniciamos el estudio dividiendo a las empresas de la muestra en dos grupos: en las que poseen certificado de calidad y en las que no lo poseen. Los valores de la variable nos señalan que 55 observaciones $(27,5 \%)$ de nuestra muestra poseen certificado, mientras que los otros $145(72,5 \%)$ carecen de él.

Con la finalidad de ganar en claridad en el estudio de los datos, no hemos considerado el tipo del certificado, limitándonos a conocer si la empresa lo posee o no. Todas las empresas que poseen dicho certificado tienen el ISO 9001:2000.

De los resultados obtenidos en los estadísticos descriptivos, observamos que, en términos generales, las empresas que poseen certificado de calidad imparten más formación (tabla 2).

Para ver si esa diferencia es significativa, hemos realizado un contraste que pretende probar la posible igualdad entre las varianzas de los dos grupos que componen nuestra muestra. De los valores obtenidos, podemos afirmar que las varianzas no son significativamente diferentes en los dos grupos, ya que el nivel de significación, utilizando el contraste de Levene, es del 25\% (tabla 3), muy superior al 5\% necesario para aceptar que las varianzas 
de los dos grupos son homogéneas; por tanto, se prueba la homogeneidad de varianzas.

Tabla 2

Análisis de la varianza sobre los valores de la variable posesión de certificados de calidad para cada grupo (estadísticos descriptivos)

\begin{tabular}{|c|c|c|c|c|c|c|c|c|}
\hline & \multirow[t]{2}{*}{$\mathbf{N}$} & \multirow[t]{2}{*}{ Media } & \multirow[t]{2}{*}{$\begin{array}{l}\text { Desviación } \\
\text { típica }\end{array}$} & \multirow[t]{2}{*}{$\begin{array}{l}\text { Error } \\
\text { Típico }\end{array}$} & \multicolumn{2}{|c|}{$\begin{array}{c}\text { Intervalo de } \\
\text { confianza para la } \\
\text { media al } 95 \%\end{array}$} & \multirow[t]{2}{*}{ Mínimo } & \multirow[t]{2}{*}{ Máximo } \\
\hline & & & & & $\begin{array}{l}\text { Limite } \\
\text { inferior }\end{array}$ & $\begin{array}{c}\text { Limite } \\
\text { superior }\end{array}$ & & \\
\hline NO & 145 & $-0,4819$ & 0,5672 & 0,0471 & $-0,5750$ & $-0,3888$ & $-1,3720$ & 1,2189 \\
\hline sí & 55 & 1,2705 & 0,5965 & 0,0939 & 1,0822 & 1,4588 & 0,0196 & 2,1828 \\
\hline TOTAL & 200 & 0,0000 & 1,0000 & 0,6999 & $-0,1380$ & 0,1380 & $-1,3720$ & 2,1828 \\
\hline
\end{tabular}

Fuente: Elaboración propia.

Tabla 3

Análisis de la varianza sobre los valores de la variable posesión de certificados de calidad para cada grupo (prueba de homogeneidad de varianzas)

\begin{tabular}{cccc}
\hline Estadístico de Levene & gl1 & Gl2 & Sig. \\
\hline 2,105 & 1 & 198 & 0,250 \\
\hline
\end{tabular}

Fuente: Elaboración propia.

Por último, el análisis de varianza nos muestra que las medias de los grupos son diferentes, puesto que el nivel de significación asociado al estadístico $\mathrm{F}$ es inferior al 1\% (tabla 4).

Tabla 4

Análisis de la varianza sobre los valores de la variable posesión de certificados de calidad para cada grupo (ANOVA)

\begin{tabular}{cccccc}
\hline & $\begin{array}{c}\text { Suma de } \\
\text { cuadrados }\end{array}$ & GI & $\begin{array}{c}\text { Media } \\
\text { cuadrática }\end{array}$ & F & Sig. \\
\hline Inter-grupos & 122,461 & 1 & 122,461 & 334,262 & 0,000 \\
Intra-grupos & 72,540 & 198 & 0,366 & & \\
Total & 195,001 & 199 & & & \\
\hline
\end{tabular}

Fuente: Elaboración propia. 
El papel de la formación de personal en el proceso de implantación de un sistema de calidad total

\section{Discusión y conclusiones}

Los sistemas de calidad total se apoyan en la participación de las personas; de esta manera, una empresa que desee implantar una filosofía de calidad total debe poner especial énfasis en la formación de sus recursos humanos.

La bibliografía especializada apoya esta idea, pero a menudo lo hace desde planteamientos puramente teóricos o desde el análisis de casos, ya que existe poca evidencia empírica de tal relación.

Con el objeto de contribuir a paliar esta carencia, nuestro trabajo ha planteado como hipótesis la relación entre calidad total y esfuerzo realizado en formación, contrastando la misma en una muestra de un sector de servicios cuya actividad es intensiva en mano de obra.

Se ha puesto especial cuidado en la medición del esfuerzo en formación dado que en la mayor parte de los estudios éste se mide de forma excesivamente simplista, pues se centra en la mayor parte de los casos en un indicador único, que no cubre el conjunto de dimensiones que pueden diferenciarse en el esfuerzo en formación.

Por tal motivo, hemos planteado una metodología que nos ha permitido cuantificar tres dimensiones distintas: la variedad de la formación ofrecida a los empleados, el tiempo dedicado por los empleados a su formación y el coste económico directo para la empresa. El indicador lo hemos hecho operativo realizando un análisis factorial a través del método de componentes principales. Los resultados obtenidos con el mismo son altamente satisfactorios, pues en un único indicador del esfuerzo de formación resumimos la información obtenida con sus tres dimensiones, la cual tiene una varianza explicada del $73 \%$.

La metodología utilizada para el contraste de la hipótesis ha sido un análisis de varianza. Los resultados obtenidos en el mismo ponen de manifiesto cómo los valores del esfuerzo formador son significativamente diferentes, en términos medios, en los dos grupos considerados; éstos son superiores para las empresas que poseen certificados de calidad.

Los resultados del trabajo tienen implicaciones tanto a nivel teórico, pues contribuye con nueva evidencia empírica a demostrar la existencia de tal relación; como a nivel práctico, para los responsables de la implantación de los sistemas de aseguramiento de la calidad en su empresa para que asuman que la filosofía que fundamenta el sistema de calidad total es la adopción de una actitud de aprendizaje continuo por parte de todo el personal y que la empresa es la que debe favorecer la misma.

Dado que el trabajo se ha centrado en un sector y un país concreto, sería interesante en un futuro buscar la generalización de resultados ampliando la población a otros sectores de servicios e incluso industriales, así como de otros contextos geográficos. 


\section{Bibliografía}

AGUILAR PASTOR, E. M. y M. A. SASTRE CASTILLO, "Diseño del trabajo y recursos humanos en un sistema de producción ajustada" en Revista de Trabajo y Seguridad Social, núm. 180, marzo 1998, pp. 151-178.

ALONSO, V. y A. BLANCO, Dirigir con calidad total, Madrid: Esic, 1990.

AMAT, J. M., Control de gestión, Barcelona: Gestión 2000, 1998.

ANTHONY, R. N., El control de gestión: marco, entorno y proceso. Vizcaya: Deusto, 2a. edición, 1997.

ARTER, D., Auditorías de calidad. Madrid: Díaz de Santos, 1993.

ARTHUR, J. B., "Effects of human resource systems on manufacturing performance and turnover”, en Academy of Management Journal, vol. 37, núm. 3, junio 1994, pp. 670-687.

AVERY, C., The Quality Management Sourcebook. Londres: Routledge, 1997.

BABSON, S., Lean Work: Empowerment and Exploitation in the Global Auto Industry, Detroit: Wayne, 1995.

BAILEY, T., "Organizational Innovation in the Apparel Industry", en Industrial Relations, vol. 32, núm. 1, invierno 1993, pp. 30-48.

BAYO MORIONES, A. y J. MERINO DÍAZ DE CERIO, "Las prácticas de recursos humanos de alto compromiso: un estudio de los factores que influyen sobre su adopción en la industria española", en Cuadernos de Economía y Dirección de la Empresa, núm. 12, mayo-agosto 2002, pp. 227-246.

BLACK, S. E. y L. M. LYNCH, "How to Compete: The Impact of Workplace Practices and Information Technology on Productivity", en The Review of Economics and Statistics, vol. 83, núm. 3, agosto 2001, pp. 434-445.

BROCHA, B. y M. S. BROCHA, Gestión de calidad. Buenos Aires: Vergara, 1992.

CROSBY, P. B., La calidad no cuesta. México: Mc Graw Hill, 1989.

DANVILA DEL VALLE, I. y M. A. SASTRE CASTILLO, "Problemas y propuestas de medición de la formación en la empresa", en Cuadernos de Estudios Empresariales, núm. 15, 2005, pp. 27-45.

DEAN, J. W. y J. R. EVANS, Total Quality: Management, Organization and Strategy, St. Paul: West, 1994.

DELERY, J. E. y D. H. DOTY, "Models of Theorizing in Strategic Human Resource Management: Tests of Universalistic, Contingency, and Configurationally Performance Predictions", en Academy of Management Journal, vol. 39, núm. 4, agosto 1996, pp. 802-835. 
DEMING, W. E., Calidad, productividad y competitividad. La salida de la crisis, Madrid: Díaz de Santos, 1989.

DRUCKER, P. F., El ejecutivo eficaz, Barcelona: Edhasa, 1992.

DRUMMOND, H., ¿Qué es hoy la calidad total?. El movimiento de la calidad, Vizcaya: Deusto, 1994.

DUNLOP, J. y D. WEIL, "Diffusion and Performance of Modular Production in the U.S. Apparel Industry”, en Industrial Relations, vol. 35, núm. 3, julio 1996, pp. 334-354.

FEIGENBAUM, A. V., Control total de la calidad, México: CECSA, 1986.

GALGANO, A., Calidad total (clave estratégica para la competitividad de la empresa), Madrid: Díaz de Santos, 1993.

GEORGE, S. y A. WEIMERSPIRCH, Total Quality Management. Strategies and Techniques Proven at Today's most Successful Companies, Nueva York: Wiley, 1994.

GROOCOCK, J. M., La cadena de la calidad, Madrid: Díaz de Santos, 1993.

HILL, C. W. L. y G. R. JONES, Administración estratégica. Un enfoque integrado, Santa Fe de Bogotá: Mc Graw Hill, 3a. edición, 1996.

ICHNIOWSKI, C., K. SHAW y G. PRENNUSHI, "The Effects of Human Resource Management Practices on Productivity”, en American Economic Review, vol. 87, núm. 3, junio 1997, pp. 291-313.

IM, J. H., S. J. HARTMAN, y P. J. BONDI, "How do JIT Systems Affect Human Resource Management?", en Production and Inventory Management Journal, vol. 35, núm. 1, enero-marzo 1994, pp. 1-4.

ISHIKAWA, K., Introducción al control de calidad, Madrid: Díaz de Santos, 1985.

, ¿Qué es el control total de calidad? (La modalidad japonesa), Barcelona: Norma, 8a. edición, 1989.

JACOBS, J., "Lean Production and Training: The Case of a Japanese Supplier Firm", en S. BABSON, Lean Work: Empowerment and Exploitation in the Global Auto Industry, Detroit: Wayne, 1995.

JURAN, M., Juran y la planificación de la calidad, Madrid: Díaz de Santos, 1990.

KELLEY, M., "Information Technology and Productivity: the Elusive Connection", en Management Science, vol. 40, núm. 3, marzo 1994, pp. 1406-1425.

, "Participative Bureaucracy and Productivity in the Machined Products Sector”, en Industrial Relations, vol. 35, núm. 3, julio 1996, pp. 374-399. 
KROLL, M., P. WRIGHT y R. A. HEIENS, "The Contribution of Product Quality to Competitive Advantage: Impact on Systematic Variance and Unexplained Variance in Returns”, en Strategic Management Journal, vol. 20, núm. 4, abril 1999, pp. 375-384.

LABOUCHEIX, V., Tratados de la calidad total, París: Bordás, 1996.

LLORÉNS, F. J., "Una evaluación de la relación entre calidad de servicio, satisfacción y actividades del cliente", en Esic-Market, núm. 93, julio-septiembre 1996, pp. 121-132.

MCDUFFIE, J. P. y T. A. KOCHAN, "Do U.S. Firms Invest Less in Human Resources? Training in the World Auto Industry", en Industrial Relations, vol. 34, núm. 2, abril, 1995, pp. 147-168.

OPPENHEIM, B. W. y Z. H. PRZASNYSKI, “Total Quality Requires Serious Training”, en Quality Progress, vol. 32, núm. 10, octubre 1999, pp. 63-74.

PÉREZ FERNÁNDEZ DE VELASCO, J. A., Gestión de la calidad empresarial: calidad en los servicios y atención al cliente. Calidad total, Madrid: Esic Market, 1994.

PHILLIPS, J. J., Handbook of Training Evaluation and Measurements Methods, Texas: Gulf Publishing Company, 1994.

SADERRA JORBA, L., La calidad total (secreto de la industria japonesa), Barcelona: Técnicas Rede, 1994.

SHAW, J. C., Gestión de servicios. La consecución del éxito de empresas de servicios mediante el desarrollo de planes, Madrid: Díaz de Santos, 1990.

TAGUCHI, G., Introduction to off-line Quality Control, Tokyo: Japanese Standards Association, 1979.

TERPSTRA, D. E. y E. ROZELL, “The Relationship of Staffing Practices to Organizational Level Measures of Performance", en Personnel Psychology, vol. 46, núm. 1, primavera 1993, pp. 27-48.

TESTA, M. R. y L. J. SIPE, “A Systems Approach to Service Quality”, en Cornell Hotel \& Restaurant Administration Quarterly, vol. 47, núm. 1, febrero 2006, pp. 36-48.

WADDELL, D. y D. STEWART, “Training Quality Managers-Do they Practice what they Preach?", en Total Quality Management \& Business Excellence, vol. 15, núm. 8, octubre 2004, pp. 1119-1129. 